\title{
Shallow Exploration of Bangkok Clay Using Multiple Square Arrays
}

\author{
P. H. Giao and D. H. Hien \\ Geosystem Exploration and Petroleum Geoengineering Program \\ School of Engineering and Technology \\ Asian Institute of Technology
}

\begin{abstract}
This study presents on implementation of multiple square arrays, including alpha, beta, gamma in investigating microanisotropy and heterogeneity of Bangkok soft clays. Fusion of these multiple arrays was automatically performed in the field by a special set up and could help identify very well the engineering facilities with a preferential direction, e.g., a concrete water pipe buried in Bangkok clay. As the amount of data generated by automatic multiple square arrays is significant an emphasis in this study was to find out a good way to present and interpret the resistivity data measured by different square array sizes at different azimuthal angles.
\end{abstract}

\section{INTRODUCTION}

To investigate anisotropy and heterogeneity of a rock or soil mass by resistivity survey it is quite commonly to employ a special procedure known as Azimuthal Resistivity Sounding or ARS (Taylor and Framing, 1988; Schmutz et al., 2006; Bolshakov et al., 1997), in which the electrode array will be rotating at different angles or azimuths with respect to a central point, commonly considered as the measuring point, to give the apparent resistivity values of the rock or soil mass in different directions. The rotating angle commonly can be $45^{\circ}$, $30^{\circ}$ or $15^{\circ}$ or else. The ARS array can be linear or square; Examples of two linear and non-linear (square) ARS arrays are given in Fig. 1a\&b

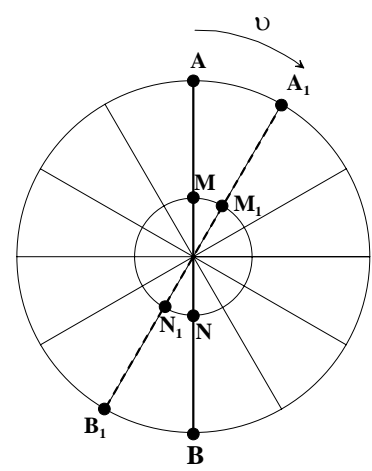

(a)

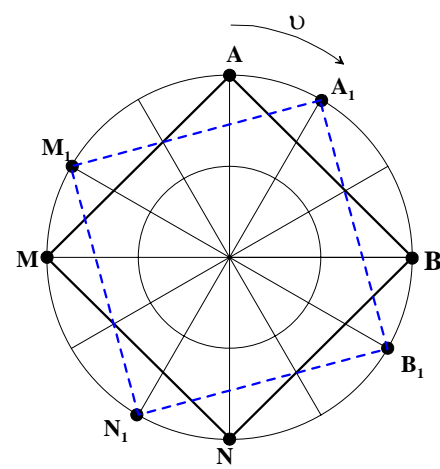

(b)
Fig. 1 (a) Linear ARS array; (b) Non-linear (square) ARS array

Taylor and Framing (1988) used Wenner and Wenner Schlumberger configurations with measurement apparent resistivity being made by rotational angles between $10^{\circ}$ to $20^{\circ}$. Watson and Baker (1999) proposed a 5-electrodes Wenner offset array for azimuthal resistivity survey. The apparent resistivity of the left-hand side four electrode $\left(\rho_{\mathrm{D} 1}\right)$ and that of the right-hand side four electrode $\left(\rho_{\mathrm{D} 2}\right)$ were introduced. The average is taken and considered as the apparent resistivity for that spacing. These two sets of measurements will be equal if the subsurface is homogeneous and isotropic. Any difference between $\rho_{\mathrm{D} 1}$ and $\rho_{\mathrm{D} 2}$ indicate the dependence of the resistivity to the anisotropic medium. Habberjam and Watkins (1967) were the first to use the square array. The advantages of this kind of array was discussed in details by Schmutz et al. (2006). The non-linear ARS array was found to be more sensitive than a linear one and had been often employed to investigate the fractured rocks (Busby and Jackson, 2006; Busby, 2000; Lane at al., 1995; Skjernaa and Jùrgensen, 1993). The azimuthal apparent-resistivity measurements used to be plotted in polar form to help depicting in which direction is developed anisotropy or heterogeneity of the investigated soil.

In the initial phase of soil exploration or site investigation for infrastructure projects developed on soft clays it is always useful if one can have information on its anisotropy. Detection of lateral heterogeneities such as the buried engineering facilities is another important task, especially in the case of redevelopment projects over an old ground. Bangkok soft clays have been recently investigated by different near-surface geophysical techniques, e.g., electric imaging, seismic refraction and ground penetration radar (Giao, 2004; Giao and Kanitorn, 2004; Giao and Vichalai, 2006), among which electric imaging proved to be very useful in mapping the subsoil profile. As a continuing step, the objective of this study is to investigate anisotropy and heterogeneity of Bangkok soft clay by multiple square arrays that could be automatically setup and performed in the field at a 
fast working speed.

\section{ELECTRIC ANISOTROPY AND ORIENTATION OF THE ELECTRODE ARRAY}

Geological materials, either rocks or soils, do often exhibit anisotropy, which used to be considered on two different scales, i.e., Micro and Macro. Microanisotropy relates to the variation of resistivity with direction within one layer or lithological unit, and it is mainly resulted from orientation of elongated rock grains as symbolically shown in Fig. 2 and is a common feature in water laid sedimentary deposits such as fluvial, lacustrine deltaic and glacial outwash deposits (Kruseman and De ridder, 1994). Macroanisotropy is generally attributed to alternative layers or beds of rock. A homogeneous and isotropic unit of rock containing water, air of rock-filled fractures is also considered to exhibit macroanisotropy. In most hard rocks, fractures occur in sets with more or less well defined preferred orientation as shown in Fig. 3:

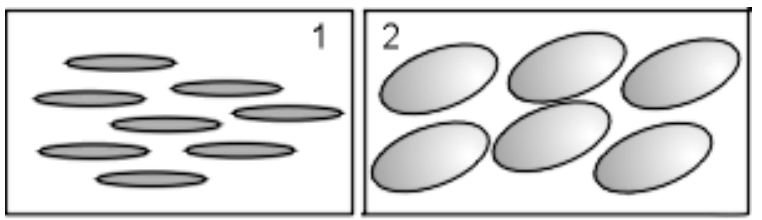

(a) Microanisotropy due to elongated (1) and flattened (2) grain orientation

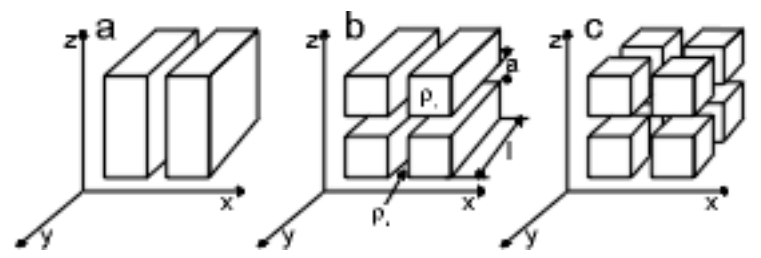

(b) Macroanisotropy due to one, two and threedirectional fracture system

Fig. 2 Microanisotropy and macroanisotrpy of geological materials (after Skejernaa and Jorgensen, 1993)

For an isotropic medium the distribution of potential due to a point source was found by Stefanescu and Schlumberger (1930), whose simplified form for a semi-infinite space is given in Eq. 1 below. The equipotential surfaces in this case are spherical.

$\mathrm{V}=\frac{\mathrm{I} \rho_{\mathrm{a}}}{2 \pi \mathrm{r}}$

Where: $\mathrm{V}$ is the electric potential at any point $\mathrm{M}$ on the surface, located at a distance $r$ from the point source with current $I$, and $\rho_{a}$ is the so-called apparent resistivity.

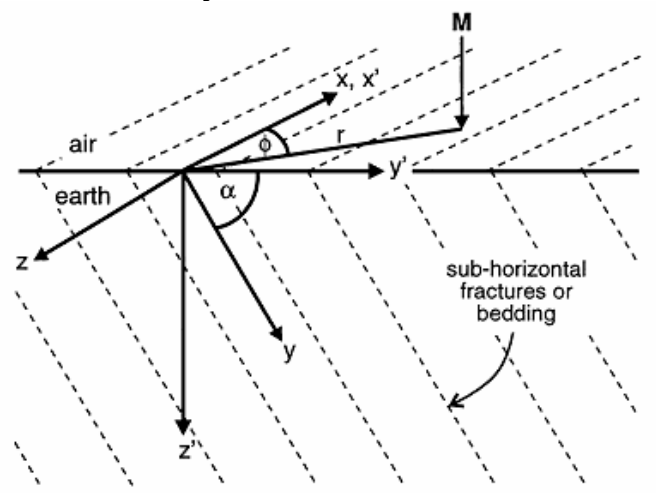

Fig. 3 A stratified or fractured rock mass (Watson and Baker, 1999)

For stratified or fractured rock types as shown in Fig. 3, the solution is more complicated as shown in Eq. 2 (Bhattachrya and Patra, 1968). The equipotential surfaces in this case are no more spherical but ellipsoidal:

$$
\mathrm{V}=\frac{\mathrm{I} \rho_{\mathrm{M}}}{2 \pi \mathrm{r}\left[1+\left(\lambda^{2}-1\right) \sin ^{2} \phi \sin ^{2} \alpha\right]^{1 / 2}}
$$

Where: $\phi$ is the angle between the fracture or bedding strike direction and the line connecting the source point and the considered point $\mathrm{M} ; \alpha$ is the dipping angle; $\lambda$ is the coefficient of anisotropy, $\rho_{\mathrm{T}}$ is the transversal apparent resistivity (perpendicular to the strike direction), $\rho_{\mathrm{L}}$ is the longitudinal apparent resistivity (parallel to the strike direction), and $\rho_{M}$ is the root means square resistivity. $\lambda$ and $\rho_{\mathrm{M}}$ are defined as follows:

$\lambda=\sqrt{\frac{\rho_{\mathrm{T}}}{\rho_{\mathrm{L}}}}$

$\rho_{\mathrm{M}}=\sqrt{\rho_{\mathrm{T}} \rho_{\mathrm{L}}}=\lambda \rho_{\mathrm{L}}=\frac{\rho_{\mathrm{T}}}{\lambda}$

For a homogeneous and isotropic medium $\lambda$ is equal to 1 . For a homogenous and anisotropic medium, $\lambda$ generally ranges between 1 and 2 because $\rho_{\mathrm{T}}$ is greater than $\rho_{\mathrm{L}}$ (Keller and Frischknecht, 1966). In the case of horizontal fracture or bedding $(\alpha=0) \mathrm{Eq}$. (2) is reduced to:

$\mathrm{V}=\frac{\mathrm{I} \rho_{\mathrm{M}}}{2 \pi \mathrm{r}}=\frac{\mathrm{I} \rho_{\mathrm{a}}}{2 \pi \mathrm{r}}$, where: $\rho_{\mathrm{a}}=\rho_{\mathrm{M}}=\lambda \cdot \rho_{\mathrm{L}}$

Thus, above horizontal fractures or flat-lying beds, the measured apparent resistivity $\rho_{\mathrm{a}}$ is larger than the longitudinal resistivity by ratio $\lambda$ (Keller and 
Frischknecht, 1966). In this case the surface azithumal resistivity survey cannot detect the horizontal anisotropy at either micro or macro scale because resulting equipotentials line will shape as circles of different radii about the central point.

In the case of vertical beds or fractures $(\alpha=\pi / 2)$ and the array orientation is parallel to the strike direction $(\phi=0)$ Eq. (2) reduces to Eq. (4b) and the measured apparent resistivity will be equal to root mean square resistivity:

$$
\mathrm{V}=\frac{\mathrm{I} \rho_{\mathrm{M}}}{2 \pi \mathrm{r}}=\frac{\mathrm{I} \rho_{\mathrm{a}}}{2 \pi \mathrm{r}} \text {, where: } \rho_{\mathrm{a}}=\rho_{\mathrm{M}}=\sqrt{\rho_{\mathrm{T}} \cdot \rho_{\mathrm{L}}}
$$

In the case of vertical beds or fractures $(\alpha=\pi / 2)$ and the array orientation is perpendicular to the strike direction $(\phi=\pi / 2)$ Eq. (2) reduces to Eq. (4c) and the measured apparent resistivity will be equal to the longitudinal resistivity, but not transverse resistivity as one might expect:

$\mathrm{V}=\frac{\mathrm{I} \rho_{\mathrm{M}}}{2 \pi \lambda \mathrm{r}}=\frac{\mathrm{I} \rho_{\mathrm{L}}}{2 \pi \mathrm{r}}$, where: $\rho_{\mathrm{a}}=\rho_{\mathrm{M}}=\frac{\rho_{\mathrm{L}}}{\lambda}$

\section{SQUARE ARRAYS}

The square array was originally developed as an alternative to Wenner or Schlumberger arrays when a dipping subsurface, bedding, or foliation was present (Habberjam and Watkins, 1967). A complete discussion of the square array and methods of data analysis is provided by Habberjam (1979). Techniques for analyzing directionalresistivity data provided by the square-array method have been developed by Habberjam (1972) and modified by Lane (1995), and namely, for each square, three measurements are made - two perpendicular measurements $(\alpha$ and $\beta$ ) and one diagonal measurement $(\gamma)$ as shown in Fig. 4.
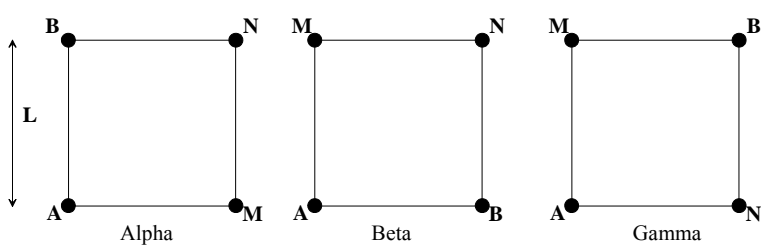

Fig. 4 Electrode positions for square array measurements (after Lane, 1995)

The apparent resistivity measured by a square array $\left(\rho_{\mathrm{a}}\right)$ is calculated in an usual as follows:

$\rho_{\mathrm{a}}=\mathrm{K} \frac{\Delta \mathrm{V}}{\mathrm{I}}$

Where $\mathrm{K}$ is the geometric coefficient
(dimensionless),$\Delta \mathrm{V}(\mathrm{mV})$ is the difference in potential measured between two potential electrodes $\mathrm{m}$ and $\mathrm{N}$, and $\mathrm{I}(\mathrm{mA})$ is the current intensity. For the square arrays ( $\alpha$ and $\beta$ ) the geometric coefficient is: $\mathrm{K}=\frac{2 \pi \mathrm{L}}{2-\sqrt{2}}$

Where $\mathrm{L}$ is the length of the square side in meter.

The $\alpha$ and $\beta$ measurements provide information on the directional variation of the subsurface apparent resistivity $\left(\rho_{\alpha}\right)$. The azimuthal orientation of the $\alpha$ and $\beta$ measurements is that of the line connecting the current electrodes (A, B). The $\gamma$ measurement serves as a check on the accuracy of the $\alpha$ and $\beta$ measurements, and namely:

$\rho_{\mathrm{a} \gamma}=\rho_{\mathrm{a} \alpha}-\rho_{\mathrm{a} \beta}$

Where $\rho_{\mathrm{a} \alpha}, \rho_{\mathrm{a} \beta}$, and $\rho_{\mathrm{a} \gamma}$ are apparent resistivity mesuared by the $\alpha, \beta$ and $\gamma$ square arrays, respectively. For an isotropic and homogeneous medium:

$\rho_{\mathrm{a} \alpha}=\rho_{\mathrm{a} \beta}$ therefore $\rho_{\mathrm{a} \gamma}=0$,

However, for a homogeneous and anisotropic medium, the apparent resistivity by the gamma square array $\left(\rho_{\gamma}\right)$ is different from zero as determined by the difference given by Eq. (7). The concept of azithumal inhomogeneous ratio (AIR) was introduced by Habberjam and Watkins (1967) as follows:

$$
\mathrm{AIR}=\frac{\rho_{\mathrm{a} \gamma}}{\rho_{\mathrm{a} \alpha}-\rho_{\mathrm{a} \beta}}
$$

Habberjam and Watkins (1967) also demonstrated that apparent-resistivity data obtained by a square array can be converted to an equivalent reistivity by Wenner or Schlumberger arrays by the following relationship:

$\mathrm{L}=\mathrm{r}\left[\frac{2(\mathrm{r}+\mathrm{s})}{2 \mathrm{r}+\mathrm{s}}\right]$

Where $\mathrm{L}$ is square array size length; $\mathrm{r}=\mathrm{AM}$ is distance between current electrode $(\mathrm{A})$ to the nearest potential electrode $(\mathrm{M}) ; \mathrm{s}=\mathrm{MN}$ is the distance between two potential electrodes (Fig. 5).
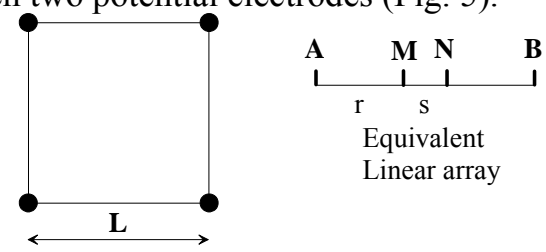

Fig. 5 Conversion of a square into an equivalent linear array (Lane et al., 1995)

Based on Eq. (9) the equivalent investigation depth of a square array comparing to a Wenner array is 
estimated as shown in Table 1:

Table 1 Conversion of a square array size to a equivalent Wenner array

\begin{tabular}{|c|c|c|}
\hline $\begin{array}{c}\text { Square } \\
\text { size, L (m) }\end{array}$ & $\begin{array}{c}\text { Equivalent } \\
\text { Wenner, a (m) }\end{array}$ & $\begin{array}{c}\text { Depth of } \\
\text { investigation (m) }\end{array}$ \\
\hline 1.00 & 0.75 & 0.39 \\
\hline 1.41 & 1.06 & 0.55 \\
\hline 2.00 & 1.50 & 0.78 \\
\hline 2.82 & 2.12 & 1.10 \\
\hline
\end{tabular}

With the apparent resistivity values obtained from alpha and beta square array measurements, the mean apparent resistivity $\rho_{\mathrm{m}}$ can be determined (Habberjam and Watkins, 1967) as follows:

$\rho_{\mathrm{m}}=\sqrt{\rho_{\mathrm{a} \alpha} \rho_{\mathrm{a} \beta}}$

Habberjam (1972) derived the expression for the variation of apparent resistivity with square-array orientation over a homogeneous anisotropic earth. For fractured rock that approximates such a medium, the predicted square-array apparent resistivity in a given orientation is:

$$
\begin{aligned}
& \rho_{\mathrm{a}}=\frac{\rho_{\mathrm{m}}}{2-\sqrt{2}}\left(\frac{2}{\sqrt{1-\left(\mathrm{N}^{2}-1\right) \cos ^{2} \phi}}-\right. \\
& \frac{1}{\sqrt{2+\left(\mathrm{N}^{2}-1\right)(1+\sin 2 \phi)}}- \\
& \left.\frac{1}{\sqrt{2+\left(\mathrm{N}^{2}-1\right)(1-\sin 2 \phi)}}\right)
\end{aligned}
$$

Where $\phi$ has the meaning as explained in Fig. 3, N is the effective vertical anisotropy calculated by the following equation:

$\mathrm{N}=\sqrt{\left(1+\left(\lambda^{2}-1\right) \sin ^{2} \alpha\right.}$

The square array has been shown to be more sensitive to anisotropy than the Schlumberger or Wenner array (Habberjam, 1972; LeMasne, 1979; Darboux-Afouda and Louis, 1989). For the square array, the anisotropic coefficient, $\lambda_{\mathrm{a}}$, is calculated as follows:

$\lambda_{\mathrm{a}}=\frac{\lambda\left(\sqrt{\lambda^{2}+1}-1\right)}{\sqrt{\lambda^{2}+1}-\lambda}$

Where $\lambda$ is anisotropy from Eq. (3a). The apparent anisotropy of the square array was compared with that by a Wenner-Schlumberger array for a true rock anisotropy by Darboux-Afoda and Louis (1989) who claimed that the former is much more sensitive to anisotropy than the latter as shown in Fig. 6.

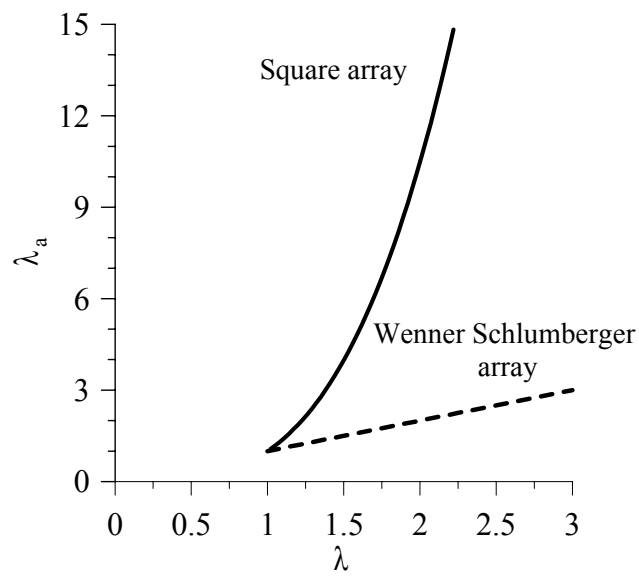

Fig. 6 Anisotropy by square array and WennerSchlumberger array (Darboux-Afoda and Louis, 1989)

\section{INVESTIGATION OF ANISOTROPY AND HETEROGENEITY OF BANGKOK CLAY}

\section{a. The Bangkok clay}

Fig. 7 shows the Bangkok clay profile at the AIT campus, consisting of weathered clay, soft to very soft clay, medium clay and part of the stiff clay layer. The investigation in this study is mainly aimed at the weathered clay, in which the facilities are buried and often need to be detected for infrastructure redevelopment projects.

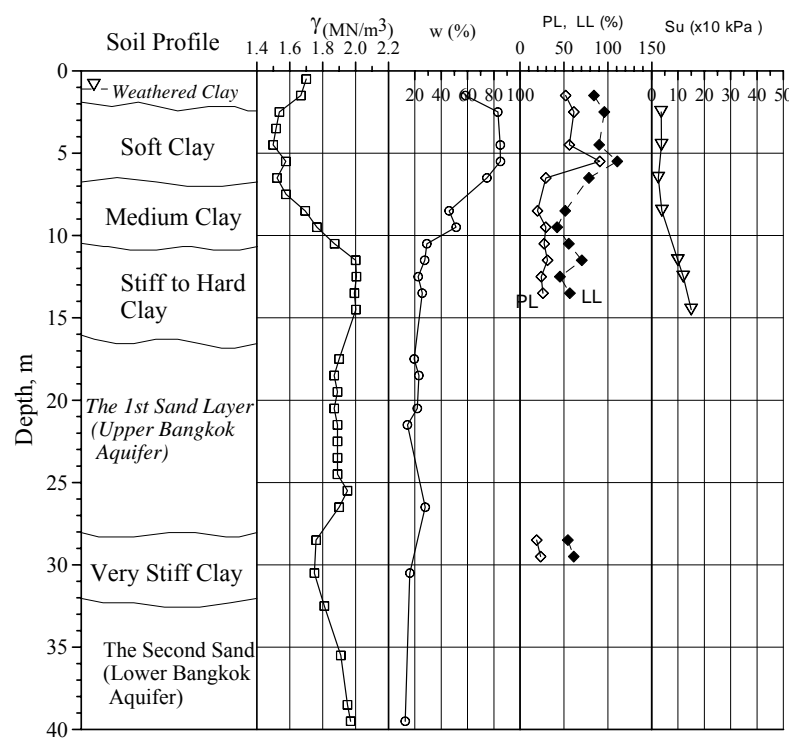

Fig.7 Soil profile at AIT campus (Giao, 2004) 


\section{b. Field set up of the multiple square arrays}

The multiple measurements were performed using the automatic resistivimeter SYSCAL R1+, two 24channel cables and 48 electrodes. Four sets of square arrays are designed with the square size increasing by $\sqrt{2}$, from $1, \sqrt{2}, 2$ to $2 \sqrt{2} \mathrm{~m}$, respectively. 48 electrodes are arranged on four circles. Each circle has 12 electrodes, located $30^{\circ}$ from each other as shown in Fig. 8a. A square array is a combination of 4 electrodes and such a grouping can make up three configurations, i.e., alpha, beta and gamma. Say, the first square array consists of electrodes 1, 4, 7 and 10, after three measurements at this positions are done corresponding to alpha, beta and gamma configurations, the electrodes are switched further to make the second square array (i.e., 2, 5, 8 and 11) and so on until the last square on the first circle (see Fig. 8b). Then the measurements will be shifted to the second, third and fourth circle levels, respectively. This set of 30degree measurements has a total of $6 \times 3 \times 4=72$ points. As we wanted to make the measurements on each 10-degree direction, the whole set up and measurements mentioned above had to repeat from a new starting direction that is 10 from the first one. Three sets of 30-degree measurements were needed and the overall amount of square array points is $72 \mathrm{x}$ $3=216$ points. The measuring sequence was setup in the Syscal environment, using software Electre II, then uploaded to Syscal $\mathrm{R}+1$.

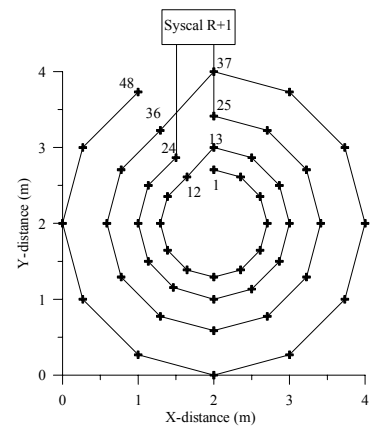

Fig. 8a Plain view of electrodes arrangement

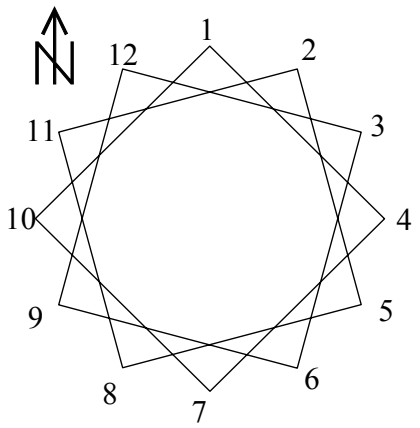

Fig. 8b Plain view of $1 \mathrm{~m}$ square array size length
In this study the multiple square array measurements were conducted at 3 points. Only the results from two points with azimuthal angle of $10^{\circ}$ are considered in this paper, i.e., one on the concrete water pipe location (point 1) and another one located about $6 \mathrm{~m}$ far from it (point 3 ) as shown in Fig. 9

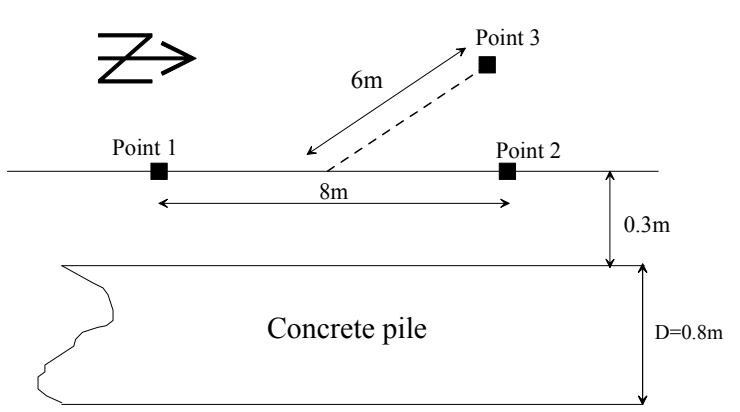

Fig. 9 Location of site investigation

\section{c. Presentation and Analysis of Data from Multiple Square Arrays}

The apparent resistivity of azimuthal resistivity survey used to be plotted in a polar diagram in order to find out the anisotropic direction corresponding to the direction of major axis of the resistive ellipsoid. The investigation point of each square array is considered at the center of square (Lane et al., 1998; Taylor and Frame, 1998; Busby, 2000\&2006). As mentioned earlier the square has been used only for fractured or bedded rocks that show a very clear anisotropy direction. The objective in this study is totally different, and namely, is to apply the multiple square arrays to study the anisotropy and heterogeneity of a deltaic clay that does not show any visible anisotropy. The results of the ARS electric imaging using multiple square arrays at the study points are presented in Fig. 10 and Fig. 11. The plotted value is the mean apparent resistivity of the square array system calculated from the values of the alpha and beta square arrays based on Eq. 11 .

Fig. 10 was made using the traditional approach with the polar diagram, in which the mean apparent resistivity is referred to the center point and comes from the same set of alpha and beta square arrays. Fig. 10a is for point 3, while Fig. $10 \mathrm{~b}$ is for point 1 . From these figures it is very difficult to find out the predominant direction of the resistivity distribution for various azimuthal measurements. In addition, the concrete pipe and its extension direction could not be detected at all. At point 3 (Fig. 10a) that it is far from the water pipe, the mean apparent resistivity values, $\rho_{\mathrm{m}}$, shows nearly perfect cycles for all of the square sizes corresponding to different depths of investigation, which implies that the vertical isotropic coefficient of subsoil is equal to 1 or in other word the soil is isotropic. At point 1 (Fig. $10 \mathrm{~b})$ the effect of the concrete pipe seems to affect and distort the circles of mean apparent resistivity.

Fig. 11 was constructed in such a way to see if the 
multiple square arrays could detect a heterogeneity buried in a homogenous ground. The main difference from Fig. 10 consists in the calculation of the mean apparent resistivity of the square array, in which the position of the measurement is considered as the middle of MN but not the center of the square array. Consequently, the mean values of apparent resistivity, calculated by means of Eq. 11, in this case would represent the average value for a soil space equal to each arc sector of $10^{\circ}$ around the center of the square. They are then plotted as separate rose diagrams corresponding to different square sizes or depths of investigation (see Table 1), i.e., $\mathrm{L}=1.0,1.41,2.0$ and $2.82 \mathrm{~m}$, respectively. It is interesting to notice that while the azimuthal resistivity diagrams for point 1 as shown in Fig. 10b do not show any preferential direction, the new presentation of the same data as plotted in Fig. 11b show clearly the extension direction of the concrete water pipe in the N-S direction at the big square sizes of $\mathrm{L}=2.0$ and $2.82 \mathrm{~m}$.

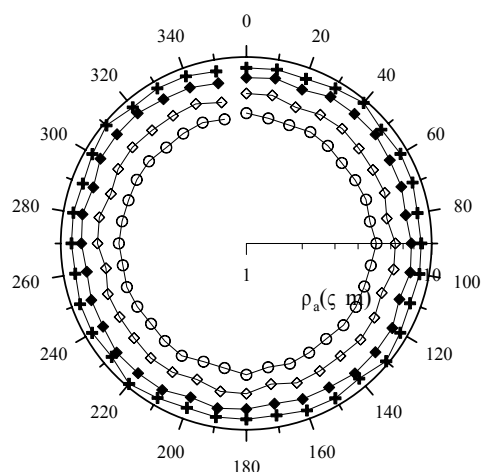

(a)

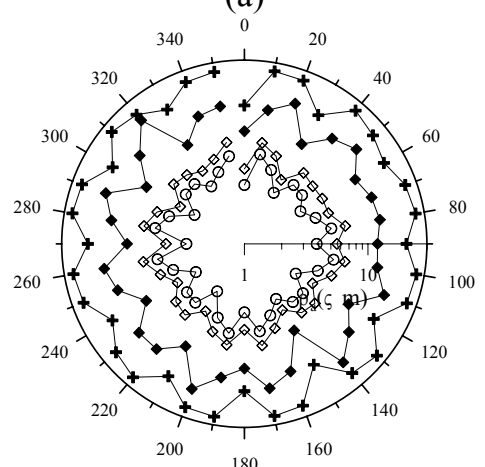

(b)

+ Square size, $\mathrm{L}=1 \mathrm{~m} \quad \diamond$ Square size, $\mathrm{L}=2 \mathrm{~m}$

- Square size, $\mathrm{L}=1.41 \mathrm{~m} \odot$ Square size, $\mathrm{L}=2.82 \mathrm{~m}$

Fig. 10 Mean apparent resistibility at (a) point 1 and (b) point 3
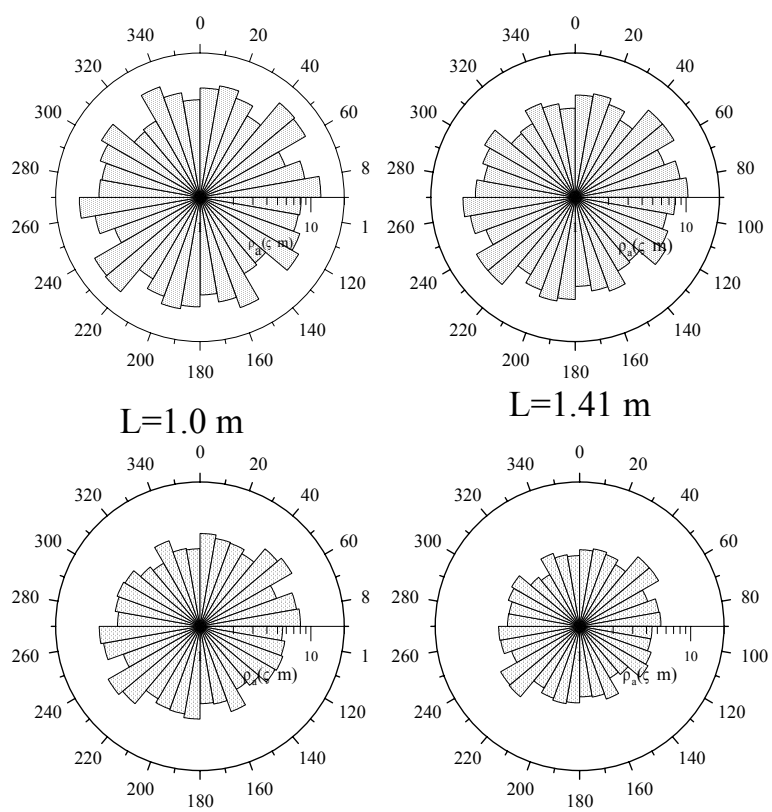

$\mathrm{L}=1.41 \mathrm{~m}$

$\mathrm{L}=2.0 \mathrm{~m}$

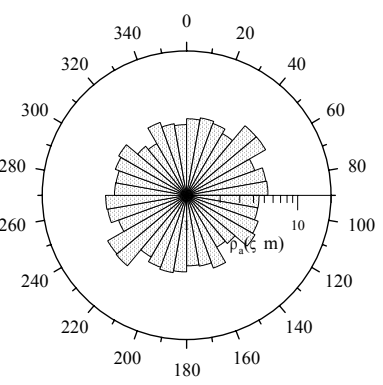

(a) Point 3: outside the concrete pipe
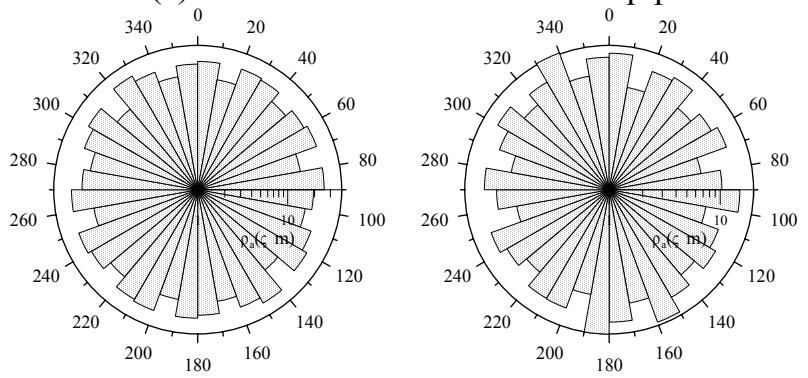

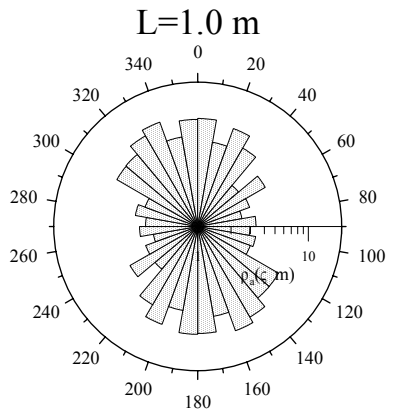

$\mathrm{L}=2.0 \mathrm{~m}$

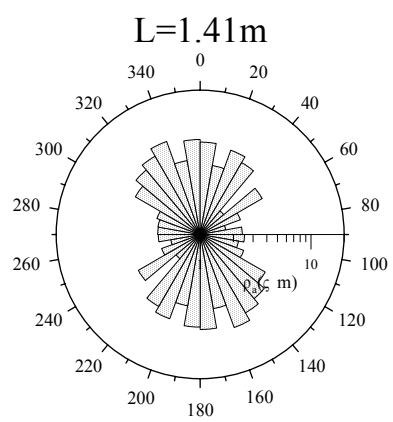

$\mathrm{L}=2.82 \mathrm{~m}$ (b) Point 1: above the concrete pipe

Fig. 11 Rose diagram of mean apparent resistivity at (a) point 3 and (b) point 1 for different square sizes.

Coming back to the topic of microanisotropy let us look at Fig. 11a, although it shows no preferential direction related to the investigated concrete water pipe it seems to suggest of a possible microanisotropy of the studied clay by the shape change of the rose diagram from almost circular (isotropic) for $\mathrm{L}=1.0$ to more elliptic with major axis oriented at $45^{0}$ for $\mathrm{L}=1.41,2.0$ and $2.82 \mathrm{~m}$. We may 
recall the earlier discussion on microanisotropy and macroanisotropy of geological materials and the conclusion here is that the weathered Bangkok clay exhibits a certain electric microanisotropy, which was not revealed by data presentation using the conventional way as done in Fig. 10a. To further investigate microanisotropy of Bangkok clay, a modified anisotropy coefficient of the square array was calculated as the root square of the ratio between a resistivity taken in any direction to the minimum value (considered to be the perpendicular to the particle arrangement direction) and plotted in Fig. 12 for different square array sizes, which shows a changing range of the anisotropy from 1.0 to 2.3 (Fig. 12a), in two main directions at $45^{\circ}$ and $135^{\circ}$ (Fig. 12b). If we consider this deltaic clay layer similar to a horizontally-layered medium the apparent resistivity $\left(\rho_{\mathrm{a}}\right)$ in this case as determined from Eq. $4 \mathrm{a}$ relates with the true longitudinal resistivity $\left(\rho_{\mathrm{L}}\right)$ by the coefficient of anisotropy $(\lambda)$.

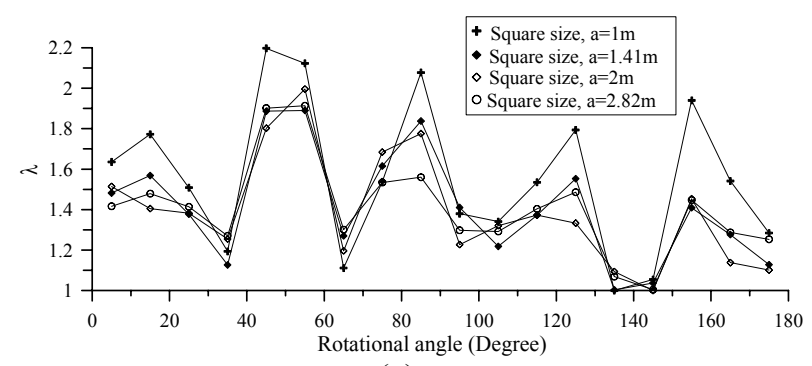

(a)

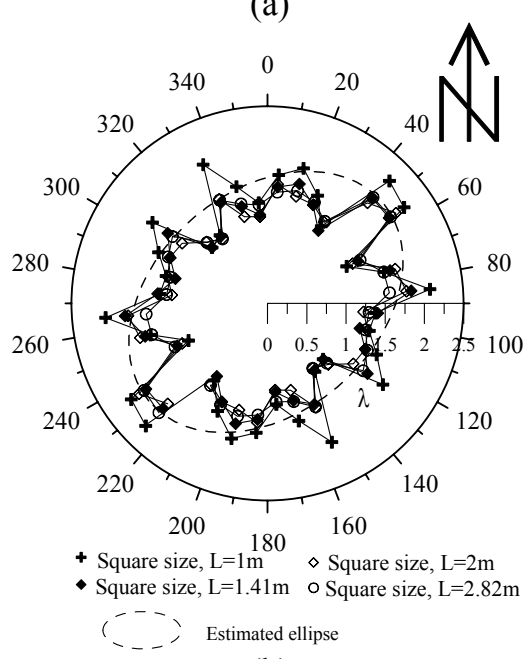

(b)

Fig. 12 The microanisotropic coefficients of the weathered soft Bangkok clay: (a) in linear presentation and (b) in polar presentation.

All of the results mentioned above may help to expand the application of square arrays for study of clay anisotropy as most applications so far have been related to macroanisotropy of rocks caused by fracturing or bedding surfaces.

\section{RESULT DISCUSSIONS}

1) The automatic multiple square arrays were successfully setup and performed in investigation of anisotropy and heterogeneity of Bangkok clay at shallow depth.

2) As the amount of data generated by fusion of multiple square arrays is quite large new procedures for data analysis and presentation were employed. The rose diagrams of the mean apparent resistivity that is assigned to the middle of $\mathrm{MN}$ instead of the square array center as conventionally done could help better to detect the development direction of a heterogeneity in Bangkok clay, which is a buried concrete pipe in this case.

3) The multiple square arrays also could assist in detecting the electric microanisotropy of the Bangkok clay, whose main directions are $45^{\circ}$ and $135^{\circ}$ to the North direction. This microanisotropy is considered to be caused by the arrangement of clay particles.

4) It was found out that many relationships related to square arrays that were proposed for study of macroanisotropy of stratified or fractured rock need to be revised and modified for a similar study on the horizontally-layered deltaic sedimentary soils.

5) Our next step is to investigate deeper targets using these multiple square arrays in a combination with other types of ARS arrays.

\section{REFERENCES}

1) Bhattacharya, B. B. and Patra, H. P. 1968. Direct methods in geoelectric souding. Elsevier Press. Amsterdam, Oxford, New York

2) Bolshakov, D.K., Modin, I.N., Pervago, E.V., Shevnin, V.A. 1998. New step in anisotropy studies : arrow-type arrays. Proceedings of the $4^{\text {th }}$ EEGS-European Section Meeting, Barcelona, Spain, pp.857-860.

3) Bolshakov D.K., Modin I.N., Pervago E.V., Shevnin V.A. 1997a. Separation of anisotropy and inhomogeneity influence by the spectral analysis of azimuthal resistivity diagrams. 3rd Meeting environmental and engineering geophysics. Proceedings. Aarhus, Denmark, 8-11 September 1997. P.147-150

4) Bolshakov D.K., Modin I.N., Pervago E.V., Shevnin V.A. 1997b. Separation of anisotropy and inhomogeneity influence by azimuthal resistivity diagram's analysis. Proceedings of the intl' symp. on environmental and engineering geophysics. Chengdu, China, pp. 239-245.

5) Busby J. and Jackson P. 2006. The application of time-lapse azimuthal apparent resistivity measurements for the prediction of coastal cliff 
failure. Journal of Applied Geophysics, 59, 261272.

6) Busby. J. P. 2000. The effectiveness of azimuthal apparent resistivity measurement as a method for determinin fracture strike orientation, Geophysical Prospecting. V 48, pp 677-695.

7) Darboux-Afouda, R., and Louis, P. 1989. Contribution des mesures de l'anisotropic electrique la recherche des aquifres de fracture en milieu cristallin au Benin. Geophysical Prospecting. v. 37, pp. 91-105.

8) Giao P. H. 2004, Electric Imaging of Bangkok Clay for Geotechnical Purposes, Proc. of the 7th SEGJ International Symposium on Imaging Technology, 24-26 Nov. 2004, Sendai, Japan, p. 481-486.

9) Giao P. H. and K. Adisornsupawat (2004), Design and Implementation of a Semi-automatic Electric Imaging Procedure to Investigate the Soft Clay Ground at NBIA, p. 223-226, Proc. of the Intl' Symposium on Lowland Technology, Sept. 13, 2004, Bangkok, Thailand.

10) Giao P. H. and C. Vichalai (2006), Construction of a Geophysical Testing Site on Soft Clays, Proc. the 8th SEGJ Intl' Symposium - Geophysical Imaging and Interpretation, p. 277-282, The Society of Exploration Geophysicists of Japan, November 26-28, 2006, Kyoto, Japan.

11) Habberjam, G.M. 1972. The effects of anisotropy on square array resistivity measurements. Geophysical Prospecting. v. 20, pp. 249-266.

12) Habberjam, G.M. 1975. Apparent resistivity, anisotropy and strike measurements. Geophysical Prospecting. v. 23. pp. 211-247

13) Habberjam, G. M. 1979. Apparent resistivity observations and the use of square array techniques, in Saxov, S., and Flathe, H. (eds.). Geoexploration Monographs. series 1, no. 9, pp. 1152.
14) Keller, G. V., and Frischknecht, F. C. 1966. Electrical methods in geophysical prospecting. Pergamon Press. London. 519 pp.

15) Kruseman, G. D. and De ridder, N. A. 1994. Analysis and evaluation of pumping test data: Internat. Inst. For Land Reclamation and Improvement Publication 47.

16) Lane. J. W, Haeli. E. P and Watson. W. M. 1995. Use of square array direct current resistivity method to detect fracture in crystalline bedrock in New Hampshire, Groundwater. V.33, no 3, pp. 476-485

17) LeMasne, G. 1979. Applications de methodes electriques et electromagnetiques l'etude geophysique des milieus fissures. These $3 \mathrm{eme}$ cycle. USTIL Montpelier.

18) Skjernaa, L. and Jorgenssen, N. O. 1993. Detection of local fracture system by azithumal resistivity survey: Example from south Norway: Memoirs of the $24^{\text {th }}$ congress of Internat. Assn. Hydrogeologist, 662-671.

19) Stefanescu, S. S. and Schlumberger, C. 1930. Sur la distribution e'lectrique potentialle autour d'une prise de terre ponctuelle dans un terrain a couches horizontals, homogenes et isotropes. Journal Physique et la Radium I, 4, 132-140.

20) Schmutz M., Andrieux P., Bobachev A., Montori J. P. And Nasri S. 2006. Azimuthal resitivity sounding over a steeply dipping anizotropic formation. A Cse study in central Tunisia, Journal of Applied Geophysics, 60, 213224.

21) Taylor, R.W., and Fleming, A.H. 1988. Characterizing jointed systems by azimuthal resistivity surveys. Ground Water. v. 26, no. 4, pp. 464-474.

22) Watson. K. A, Baker. R. D, 1999, Differentiating anisotropy and lateral effects using azimuthal resistivity offset Wenner sounding, Geophysics. V. 64, no 3, pp 739-745 